\title{
Technical specifications of a service-oriented architecture for semantic interoperability of EHR - electronic health records
}

\author{
Edgard Costa Oliveira \\ Engineering College, \\ University of Brasilia (UnB), Brazil \\ ecosta@unb.br
}

\begin{abstract}
Many countries are in the run for implementing their national electronic health strategies, on top of the existing health systems administrative and clinical procedures. There have been international successful initiatives in these cases, however, a substantial effort is necessary in order to adapt the best practices to national realities. In Brazil, the effort of implementing a technological solution, inspired by these international cases, is under way. This paper describes the technical specification of an implementation architecture for a SOA-based infrastructure that created the Electronic Health Record solutions for the Brazilian public health system. We identified here, the context where it has been implemented and particular need of the National e-health strategy, the information health management standards and patterns used. We described the SOA architecture built and implemented, aiming at specific needs at stake, such as basic care, hospital, medicines and individual patient's information. The basic EHR profiles used are also described with an emphasis on specific use cases. The integrated solution implemented was also presented in a general design that brings together, in a simplified manner, the components of a semantically interoperable $S O A$ architecture for electronic health records designed specifically for the Brazilian health context, but we hope can be an asset and success case for other ongoing e-health strategy design initiatives.
\end{abstract}

Keywords: Electronic Health Record - EHR, Clinical information repository, Health knowledge model.

\section{INTRODUCTION}

The Brazilian Ministry of Health is on the way to implement, by the end of 2016, the national Electronic Health Record infrastructure and system. This national SOA-based 5-year project resulted in a set of information sources and services that provides a health information distributed repository for users of the whole national health system (SUS). It contains clinical information collected and shared by various clinical information systems (CIS). Particularly, this infrastructure provides information to be available at the patient's electronic records (PEPs or EHRs) in order to obtain historical information, in a safe, integrated and opportunistic information care process.

The sets of information will be available via the integration of existing information systems to the recently created National EHR (Electronic Health Record). This system is available to healthcare professionals, service providers as well as patient himself and all users of Brazilian health system - SUS. This solution provides ways to support clinical decisions, in man levels, aiming at the improvement of health services via an integrated health attention view, patient-centered and focused on the continuity of healthcare.

The benefits that the national EHR solution provides are:

1. to increase health information access;

2. to reduce time searching for health information;

3. to support clinical decision, raising efficiency and health care safety;
4. to improve healthcare continuity;

5. to raise confidence of stakeholders such as health services providers, professionals, medical team and patients.

6. to reduce information overlay and to reduce costs;

7. to increase capacity in promoting efficient public health policies.

Thus, the Brazilian Ministry of Health has been developing an interoperability clinical information systems model, described in the technical architecture of the Brazilian national electronic health record solution. This solution provides a set of health information services via a health service bus. They were conceived and developed with the support of semantic resources framework, structured in a clinical knowledge models, based on the OpenEHR. This solutions assures accuracy and security of shared clinical information even if they come from different clinical systems.

The ministry considers that the Brazilian EHR solutions is by nature a complex process that is constantly under evolution. Its services, functionalities and resources are incorporated in a scalable and iterative manner, within each projects phases. This is why the ministry is working to establish the following EHR structuring system components.

- Demographic services: a set of services that provide single users identifications in the public health system (SUS). This service was established based on the National Card Identification system.

- Health Service Bus: this is the service infrastructure created for publication, orchestration, governance and to make sure that EHR services usage policies are being followed.

- Security services: such as authentication and access control (authorization).

- Semantic Framework: consists of a set of services created specifically to disclose and manage standardized semantic resources such as terminologies and clinical knowledge models, based on the OpenEHR model. This model is mainly used when providing structure for the shared and stored clinical information.

In the present stage of the Project, we specified the services related to the Clinical Documents Repository (CDR) of our EHR. This will service uses a distributed architecture, based on the Clinical Documents Register (RegDC), which is a single and centralized indexer. This repository is capable of storing and indexing clinical information collected from structured documents based on a metadata established in the semantic framework. In particular, by specifying binds and relationships with the terminologies and information models specified by the knowledge models (templates and OpenEHR archetypes) which were then standardized and customized to be used by the Brazilian EHR. Thus, the Brazilian Ministry of Health is 
prospecting software platforms that can implement RDCs and RegDCs as well as auditing trails repositories (ATRs) compliant with the interoperability architecture defined.

\section{TECHNICAL EHR ARCHITECUTRE}

In this section, we describe the components of the electronic health record solutions designed to receive the SOA-based solution. Starting from the establishment of the context where health information is generated and used.

\subsection{Interoperability standards for health information in} Brazil

Many different health information standards were developed and adopted along the last years. In Brazil, the discussion about these patters resulted on a national act from the Ministry of Health [1] that regulates the use of the following models of information and terminologies in the Brazilian health care context.

\section{TABLE 1: INTEROPERABILITY PATTERNS FOR THE EHR} IN BRAZIL

\begin{tabular}{|l|}
\hline Web Services: SOAP, WS-Security, WSDL \\
\hline $\begin{array}{l}\text { Document Encoding: XML (based on metadata from } \\
\text { clinical information models) }\end{array}$ \\
\hline $\begin{array}{l}\text { Registry services and Clinical Documents Repository: } \\
\text { IHE - XDS.b }\end{array}$ \\
\hline $\begin{array}{l}\text { Demographic services (identification): IHE - PIXv3 } \\
\text { PDQv3 }\end{array}$ \\
\hline Clinical content: OpenEHR (clinical knowledge models) \\
\hline Terminologies: \\
\hline Clinical terms: SNOMED [6] \\
\hline Diseases classification system: CID 10 \\
\hline
\end{tabular}

Clinical procedures: Tabela Unificada do SUS (SIGTAP), TUSS, CBHPM

\begin{tabular}{l} 
Laboratory exams codes: LOINC (Logical Obs. \\
Identifiers, Names and Code) \\
\hline Primary care system: ICPC-2 (CIAP-2) \\
Image encoding: DICOM
\end{tabular}

\subsection{Clinical information systems}

The Brazilian electronic health record is gradually interoperable with the different e-Health applications and services, such as:

Personal Health Record (PHR): a clinical information system that electronically manages information from the patients personal records, based on Brazilian law [2]. PHRs are usually used within limited scope of a particular health service agent. The ministry of health develops and manages the following PHRs solutions, which are gradually being integrated to the National EHR solution.

eSUS AB: electronic personal health record used in the primary care level in Brazil.

eSUS Hospitalar: electronic personal health record used in hospitals.

eSUS Ambulatorial: electronic personal health record used in clinics of specialized attention.

Registro Eletrônico Pessoal de Saúde - REPS: it is a personal electronic health record that gives the patient access to EHR and is controlled by the patient himself. This system also allows the patient to have access to his/her information contained in the national EHR. This system will also be integrated to the following REPS developed by the Ministry of Health (as shown on figure 1).

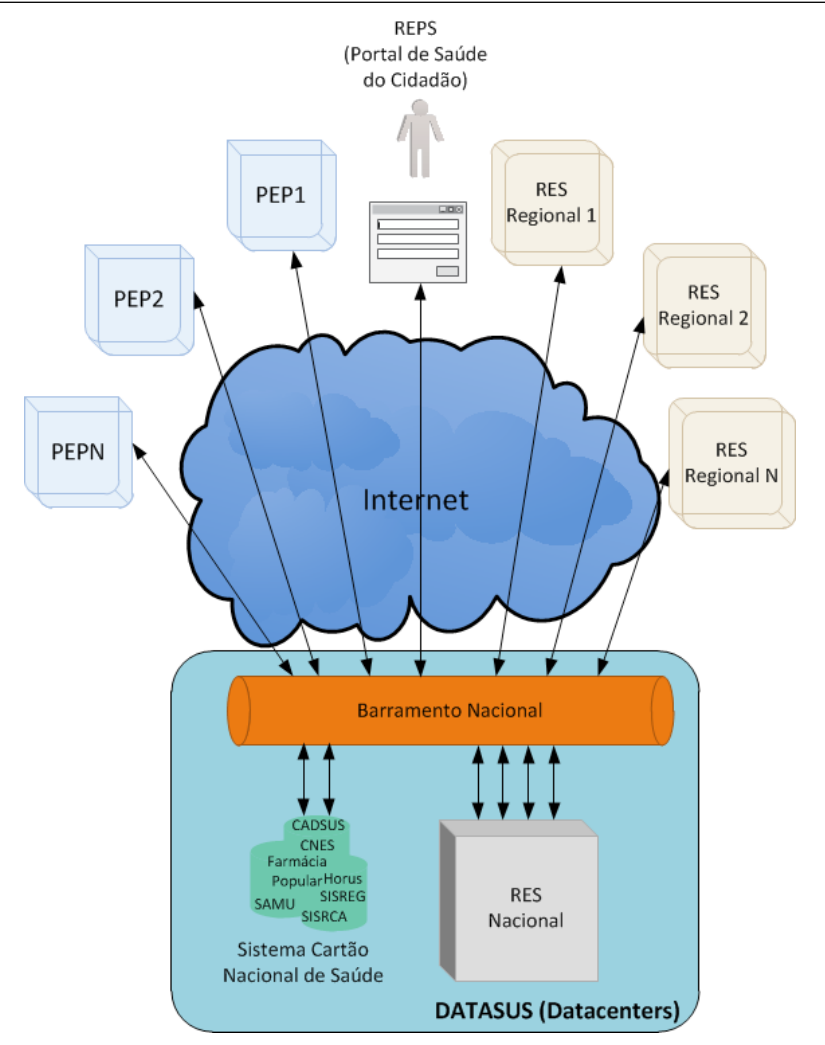

Figure 1 - E-Health Platform

CNS Digital: a mobile application to allow access to patient's health related information. br):

Citizen's Health Portal (http://portaldocidadao.saude.gov.

Users in general have access to all their health information, such as medical appointment historical, medicines acquired under the pharmacy national assistance policy as well as other personal clinical record information.

The National Electronic Health Register: a national database of all regional EHRs gathered in one general system.

E-Health services and applications are interoperable with each other and with external systems, even those that make use of different technologies or belong to other levels of service provision. This is made via a National Health Service Bus, built based on a set of internationally known interoperability standards, as we can see in figure 1 .

\section{SOA EHR ARCHITECTURE}

Based on the Brazilian standards from a national EHR [1], the technical architecture is based in the IHE - International Health Enterprise infrastructure [3] which established a set of profiles to be considered in such solutions. Figure 2 shows the profiles defined to be used in the technical infrastructure of the Brazilian National EHR.

The profiles used from the IHE framework, used in the national EHR solution, are briefly described below (a deeper specification of the profiles is provided in section 3 of this paper).

Patient Identifier Cross-referencing HL7 V3 (PIXV3) is a profile recommendation that allows crossing different user's (patients) identification domains. These patient identifiers can be used by the consuming services of these entities to relate them to the information of the same patient in different identification systems. This profile have the same functionalities of those used in the PIX with HL7v3. It uses a message standard and SOAP transportation protocol. 


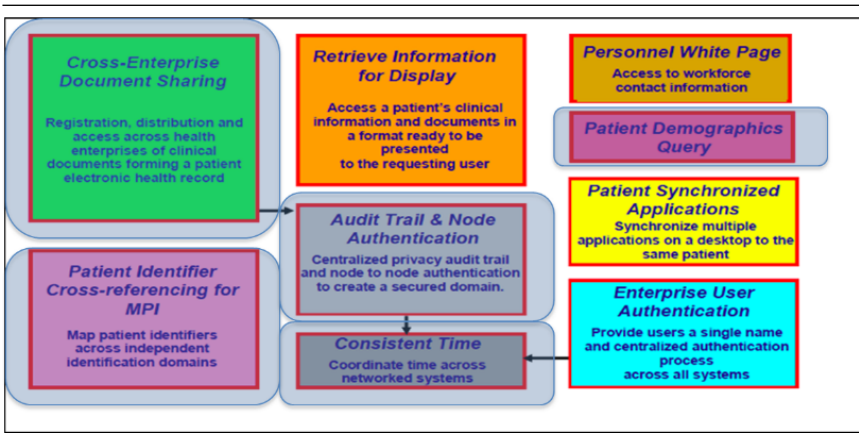

Figure 2 - IHE Profiles used in the Brazilian EHR infrastructure

Patient Demographics Query HL7 V3 (PDQV3) - these contain the same functionalities of a PDQ with HL7v3 profile, and also uses SOAP protocol for transportation.

Cross-Enterprise Document Sharing (XDS) - it allows a set of health institutions belonging to a same affinity domain (XDS Affinity Domain), for example a health care regional network, to share their patient's clinical documents. This profile describes the configuration of an ebXML registry with enough details to foster document sharing via clinical information networks.

Audit Trail and Node Authentication (ATNA) - it defines the security characteristics so that different systems, namely IHE profile network nodes, can be safely connected. In particular, this profile is used to define auditing messages between security notes and auditing repository.

Consistent Time (CT) - synchronization mechanism between different actors and systems. This is a necessary component even in different solutions such as PIX/PDQ. The synchronization medium error time is lower than 1 second.

Figure 3 presents the general architecture of the Brazilian National EHR SOA Architecture, in which we identified the following components and resources.

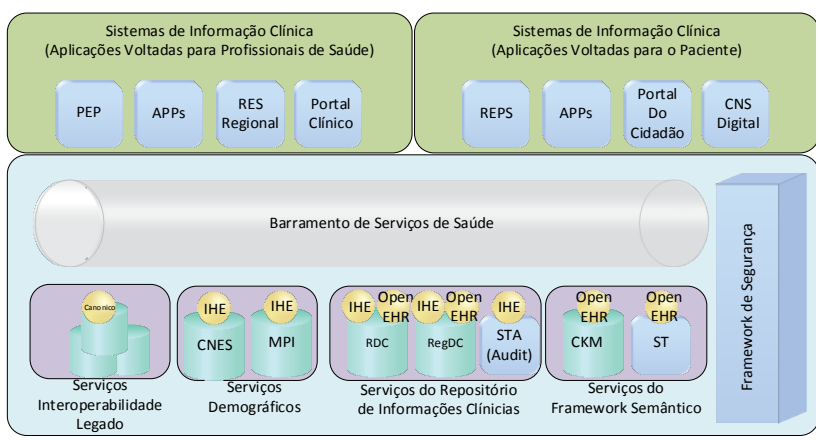

Figure 3 - SOA Architecture of the Brazilian National EHR

Demographic services - these are services implemented based on the concepts of MPI - Master Patient Index. These services offer a user's single identification service by using their unique National Health Card number. Its functionalities are: demographic requests (based on the IHE PDQv3 profile) and records updates (based on IHE Pixv3 profile). These profiles are specified here [4].

Health Service Bus - it is a service infrastructure to publish, orchestrate, govern and reassure use policies of the EHR information services, including legacy systems services.

Semantic framework services - it was built based on a two level modelling concepts, which is the dynamic implementation of both knowledge information models from the OpenEHR framework.

Clinical information repository services - it creates the clinical documents register and repository (RegDC and RDC), that supports persistence, indexing and clinical information search, in the OpenEHR format. This has been available initial via compatible services with the IHE XDS.b infrastructure from the IHE - Integrating HealthCare Enterprise. It includes the Auditing Trail Server - ATS which generates auditing trails (STA) that are related to the access to the RegDC and RDC, both implemented bade on the IHE ATNA infrastructure profile.

Security Framework - it is a set of services and resources used to guarantee that access policies and the use of RES are safely applied, including access authentication and authorization, according to the use policies and consent definitions controlled by the users of the National Health System (SUS).

\section{OPENEHR: ARCHETYPES AND TEMPLATES TECHNICAL SPECIFICATION}

In this section, we present a technical specification of the message architecture of the minimum data set (CMD) for health care, based on the OpenEHR standards. The definition of the CMD information models and its implementation in the real case of the SOA architecture was base in the archetype model of the OpenEHR. In order to provide graphic visualization or archetype and template changes or editions, the following tools are needed: Archetype Editor: archetypes visualizing and editing; Template Designer: it is a template editor and visualizer. [5]

The content of the document for the CMD (minimum data set) is the template located in the repository (file name cdms07102015.oet) which is the XMLSchema (template) created by the Template Designer.

Figure 4 shows the actors and transactions defined by the Integration Profile XDS.b.

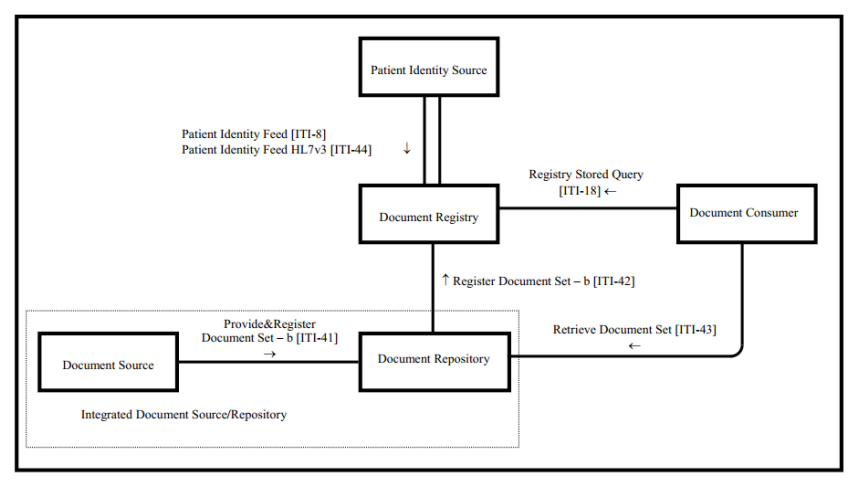

Figure 4 - Actors and transactions defined by the integration profile XDS.b

The actor Document Source is the producer and Publisher of documents that is responsible for sending documents to the actor Document Repository. It also supplies metadata to the actor Document Repository to the following register of documents by the actor Documents Registry.

Each document metadata is stored and maintained by the Documents Registry, including information about the Document Repository where it is stored. Moreover, the Document Registry answers to the documents requests mad by the actor Documents consumer, according the specific consult parameters. The Document Repository actor is both responsible for the persistent storage of this document and for its own registry in the appropriate Document Registry.

The Patient Identification Source actor is the supplier of the single identifier to each patient and maintains a collection of patient's characteristics as an identification means. This actor allows the work of Documents Registry in the single identification of patients. 


\section{IHE ATNA PROFILE - AUDIT TRAIL AND} NODE AUTHENTICATION

This profile establishes the security characteristics of different systems, called network nodes by the IHE profile, so that they can be safely connected. In this profile, we have: the security description of the environment (user's id, authentication, authorization, access control etc.); basic auditing requirements definitions; basic security communication requirements of the node, by using TLS 495 or equivalent functionality; the definition of auditing messages between safe noes and the auditing repository. This profile was defined in a way that it is possible to extend it via one of its options.

VI. Profile IHE HL7 PIX v3 - Patient Identifier Cross-referencing and Profile IHE HL7 PDQ v3 - Patient to the documents contents, any type of clinical information is supported, independently of what kind of content a format was represented. In fact, the affinity domain may contemplate policies related to the format, structure and content of shared documents. However, we hope that this repository can be a storage of documents and information in the OpenEHR format.

Figure 5 illustrates a typical scenario of integration between a patient's record and a health record, which represents the model of implementation of the Brazilian National Health Record infrastructure in practice. In this environment, we can realize the following transactions: Patient Identification (demographic services) (1), (2), (7), (8); EHR Visualization (REPS/PEP): (3), (11); Information delivery by EHR (RDC and RegDC): (4), (5), (6); Information retrieval from EHR (RDC e RegDC): $(9,10)$

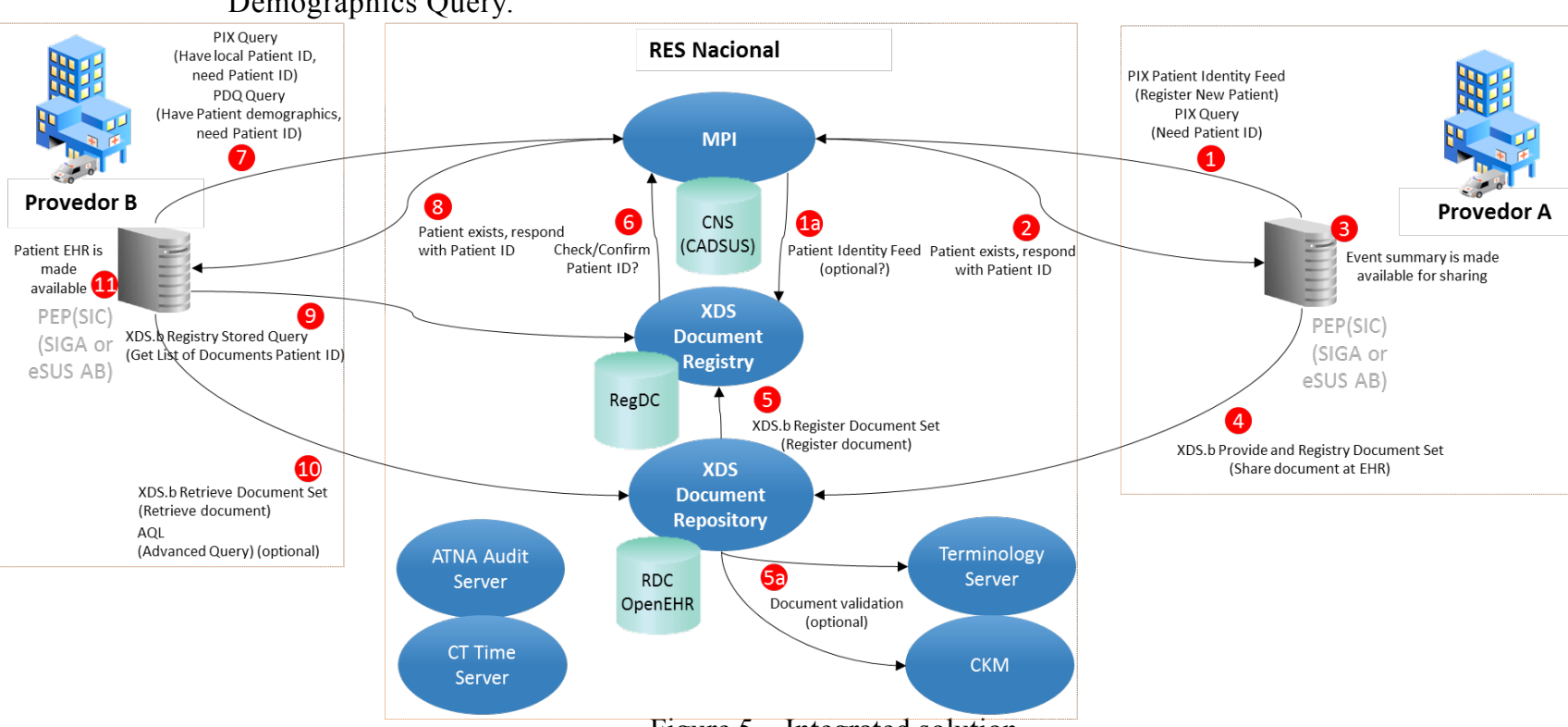

Figure 5 - Integrated solution

These profiles were implemented by the MPI into the National Health Record System (Cartão Nacional de Saúde) and are responsible for the demographic information about the patient. However, the CMD message architecture takes into account the implementation of the IHE XDS.b v3 (register and repository) and its transactions (messages). The content of a XDS message is the template created by the Template Designer (described in section 2). HL7 PIX and PDQ v3 are also used to manipulate demographic data. For security and auditing IHE ATNA is used. To store and retrieve information in the database, a message transformation from and to XSD and the database.

The integration Cross Enterprise Document Sharing (XDS.b) profile offers a standard based specification to manage document sharing between health institutions. It is based on the use of Document Repository (RDC) and Document Registry (RegDC), in order to establish a transversal information record about a patient within a given clinic domain. These two register are distinct entities with distinct responsibilities: RDC is responsible for storing documents in a transparent, secure, trustworthy and persistent manner, in order to respond the document retrieval requests. RegDC is responsible for storing information about these documents by using metadata, so that these documents can be easily found, and then selected and retrieved independently of which repository it is located at.

Documents are provide by one or more document source and are accessed by one or more document consumer. This profile considers that an affinity domain is a group of health institutions that agree in using policies and a shared infrastructure in order to work together. Once this profile has a neutral role in relation

\section{CONCLUSIONS}

This paper presented a list of specifications of the solutions implemented in the establishment of the Brazilian national electronic health record infrastructure. It brings the main functionalities, including the definition of the SOA architecture of the solution. We believe that these interoperability requirements allow the implementation of web services that are interoperable with other internal and external health systems, based on the WS-I that states the security profiles. The OSB - Oracle Service Bus infrastructure implemented in the Ministry of Health is where the services are made available and are orchestrated by it. It allowed the interoperation with the MPI via PDQ and PIXv3 transactions and implemented the XDS.b transactions.

The solutions supports and recognizes documents in the OpenEHR format, in order to store, index and retrieve files from the repository and from the clinical documents register.The storage functions were designed to store clinical data separately from demographic data. The documents and clinical information are indexed with the use of non-nominal identifiers, which can be used via the existing MPI solution.

The clinical data is stored in a database system or file system that is uncoupled from the service repository. This is why the Ministry of Health adopts the Oracle data bank version $11 \mathrm{~g}$, where it uses an equivalent database management system. In order to allow requesting and searching in the databases, the solution uses metadata parameters of collected clinical documents. Soon we can implement a query language based on OpenEHR platform called AQL. It also allows OpenEHR documents from XDS.b transactions to be stored in compliance 


\section{with this profile.}

The documents register functions allow treatment of documents taken from multiple repositories, by using the XDS.b profile. We also saw in this paper that the auditing functions allow the generation of auditing trails related to the access to the repository according to the ATNA profile. Finally, the data validation function of the solution allow the validation of OpenEHR documents, via the interaction with the semantic framework.

\section{REFERENCES}

[1] Brasil. Ministério da Saúde. Portaria 2073. 2012. Disponível em http://bvsms.saude.gov.br/bvs/ saudelegis/gm/2011/prt2073_31_08_2011.html

[2] Resolução CFM No 1821/2007. Publicada no D.O.U. de 23 nov. 2007, Seção I, pg. 252. Available at: http://www. portalmedico.org.br/resolucoes/cfm/2007/1821_2007.htm

[3] IHE e Arquitetura de Interoperabilidade do RES v.1.1

[4] Brasil. Ministério da Saúde. Catálogo de serviços. Disponível em http://datasus.saude. gov.br/interoperabilidade/catalogo-de-servicos

[5] OpeneEHR. Modelling tools. Disponível em http:// www.openehr.org/downloads/modellingtools

[6] SNOMED Clinical Terms Technical Reference Guide. International Health Terminology Standards Development Organisation, July 2016. 